\title{
Does Diabetes Really Impact on Postoperative Survival in Patients with Colorectal Cancer?
}

\author{
Hungdai Kim \\ Department of Surgery, Kangbuk Samsung Hospital, Sungkyunkwan University School of Medicine, Seoul, Korea
}

See Article on Page 424-428

According to a recent cohort study, diabetic patients had a significantly greater risk of colorectal cancer (CRC) than non-diabetic patients [1]. This study went one-step further and analyzed the postoperative survival difference between diabetic and nondiabetic patients with comorbid colorectal cancer. The authors found that no relationship existed between the presence of diabetes and the recurrence of or the survival rate for colorectal cancer. However, many other studies have reported that diabetes significantly and negatively impacts overall survival (OS) and that the cancer-specific survival (CSS) and prognostic impact of diabetes on OS and CSS were particularly significant in patients with stage II colon cancer [2]. However, many articles report results different from what we know. For instance, in patients undergoing colorectal cancer surgery, those with diabetes had a $23 \%$ lower in hospital mortality and fewer postoperative complications compared to those without diabetics [3], but the reason for this finding is uncertain.

There are many factors affecting the result of this study. An important bias is smoking. The association with colorectal cancer risk was significantly modified by smoking status [1]. Even though the presence of diabetes did not affect the overall survival in patients diagnosed with CRC, the quality of diabetes care prior to CRC diagnosis, which persisted after diagnosis, may have moderated the mortality in patients with diabetes [4]. Insulin itself, particularly insulin glargine, may play more of a mitogenic than a carcinogenic role in association with different types of cancer, suggesting an amplified rate of existing

Correspondence to: Hungdai Kim, M.D.

Department of Surgery, Kangbuk Samsung Hospital, Sungkyunkwan University School of Medicine, 108 Pyeong-dong, Jongno-gu, Seoul 110-746, Korea

Tel: +82-2-2001-2137, Fax: +82-2-2001-2131

E-mail: hungdai.kim@samsung.com

(C) 2010 The Korean Society of Coloproctology

This is an open-access article distributed under the terms of the Creative Commons Attribution NonCommercial License (http://creativecommons.org/licenses/by-nc/3.0) which permits unrestricted noncommercial use, distribution, and reproduction in any medium, provided the original work is properly cited. tumor growth in the presence of insulin analogs [5]. Thus, gaining confirmative data through a simple comparison between the presence and the absence of diabetics for estimating a prognosis for the disease is extremely difficult because it is hard to control so many kinds of biases. Furthermore, the reliability of the results is quite low because the number in the observation group was relatively small (67) compared with that in the control group (590) in this study. Therefore, a population-based prospective cohort study, rather than a case-control study, is needed to yield data in which one can have more confidence.

\section{REFERENCES}

1. He J, Stram DO, Kolonel LN, Henderson BE, Le Marchand L, Haiman CA. The association of diabetes with colorectal cancer risk: the Multiethnic Cohort. Br J Cancer 2010;103:120-6.

2. Huang YC, Lin JK, Chen WS, Lin TC, Yang SH, Jiang JK, et al. Diabetes mellitus negatively impacts survival of patients with colon cancer, particularly in stage II disease. J Cancer Res Clin Oncol 2010 Apr 13 [Epub]. DOI: 10.1007/s00432-010-0879-7.

3. Anand N, Chong CA, Chong RY, Nguyen GC. Impact of diabetes on postoperative outcomes following colon cancer surgery. J Gen Intern Med 2010;25:809-13.

4. Chiao EY, Nambi PV, Naik AD. The impact of diabetes process and outcome quality measures on overall survival in patients with co-morbid colorectal cancer. J Cancer Surviv 2010;4:381-7.

5. Call R, Grimsley M, Cadwallader L, Cialone L, Hill M, Hreish V, et al. Insulin: carcinogen or mitogen? Preclinical and clinical evidence from prostate, breast, pancreatic, and colorectal cancer research. Postgrad Med 2010;122:158-65. 